\title{
Plasma P-tau181 in Alzheimer's disease: relationship to other biomarkers, differential diagnosis, neuropathology and longitudinal progression to Alzheimer's dementia.
}

Shorena Janelidze $^{1 * \#}$, Ph.D., Niklas Mattsson ${ }^{1,2,3 \#}$, M.D., Ph.D., Sebastian Palmqvist ${ }^{1,2}$ M.D., Ph.D., Philip Insel ${ }^{1}$, M.Sc., Ruben Smith ${ }^{1,2}$, M.D., Udo Eichenlaub ${ }^{4}$, Ph.D., Henrik Zetterberg ${ }^{5,6,7,8}$, M.D., Ph.D., Kaj Blennow ${ }^{5,6}$, M.D., Ph.D., Erik Stomrud ${ }^{1,9}$, M.D., Ph.D, Jeffrey L. Dage ${ }^{10}$, Ph.D., Oskar Hansson $^{1,9^{*}}$, M.D., Ph.D.

${ }^{1}$ Clinical Memory Research Unit, Lund University, Lund, Sweden. ${ }^{2}$ Department of Neurology, Skåne University Hospital, Lund, Sweden. ${ }^{3}$ Wallenberg Center for Molecular Medicine, Lund University, Lund, Sweden. ${ }^{4}$ Roche Diagnostics GmbH, Penzberg, Germany. ${ }^{5}$ Department of Psychiatry and Neurochemistry, the Sahlgrenska Academy at the University of Gothenburg, Mölndal, Sweden. ${ }^{6}$ Clinical Neurochemistry Laboratory, Sahlgrenska University Hospital, Mölndal, Sweden. ${ }^{7}$ Department of Neurodegenerative Disease, UCL Institute of Neurology, Queen

Square, London, United Kingdom. ${ }^{8}$ UK Dementia Research Institute at UCL, London, United Kingdom. ${ }^{9}$ Memory Clinic, Skåne University Hospital, Malmö, Sweden. ${ }^{10}$ Eli Lilly and Company, Indianapolis IN 46285 U.S.A.

\section{Corresponding Authors}

Oskar Hansson (Oskar.Hansson@ @ed.lu.se), Memory Clinic, Skåne University Hospital, SE-20502 Malmö, Sweden, \& Shorena Janelidze (shorena.janelidze@med.lu.se), Clinical Memory Research Unit, Department of Clinical Sciences Malmö, Lund University, Sölvegatan 19, BMC B11, 22184 Lund, Sweden.

\#Equally contributed as first authors

\section{Word count:}

Abstract: XXX

Main text: XXX

Online-only supplements:

eMethods, eTables 1-6 and eFigures 1-3 


\begin{abstract}
Plasma phosphorylated tau181 (P-tau181) might be increased in Alzheimer's disease (AD), but its usefulness for differential diagnosis and prognosis is unclear. We studied plasma P-tau181 in three cohorts, with a total of 589 individuals, including cognitively unimpaired participants and patients with mild cognitive impairment (MCI), AD dementia and non-AD neurodegenerative diseases. Plasma P-tau181 was increased in preclinical AD and further increased at the MCI and dementia stages. It correlated with CSF P-tau 181 and predicted positive Tau positron emission tomography $(\mathrm{PET})$ scans (area under the curve $(\mathrm{AUC})=0.87-0.91$ for different brain regions). Plasma P-tau181 differentiated $\mathrm{AD}$ dementia from non-AD neurodegenerative diseases with an accuracy similar to that of Tau PET and CSF P-tau181 (AUC =0.94-0.98), and detected AD neuropathology in an autopsy-confirmed cohort. High plasma P-tau181 was associated with subsequent development of AD dementia in cognitively unimpaired and MCI subjects. In conclusion, plasma P-tau181 is a noninvasive diagnostic and prognostic biomarker of $\mathrm{AD}$, which may be useful in clinical practice and trials.
\end{abstract}




\section{INTRODUCTION}

Accumulation of misfolded $\beta$-amyloid $(\mathrm{A} \beta)$ peptides in extracellular plaques and tau protein in neurofibrillary tangles (NFT) are defining neuropathological features of Alzheimer's disease (AD). The diagnostic workup of AD has been revolutionized by cerebrospinal fluid (CSF) and positron emission tomography (PET) biomarkers, which allow researchers, clinicians, and drug developers to identify $\mathrm{AD}$ in living humans with much greater precision than by clinical testing alone (Jack et al. 2018). The core CSF biomarkers include $A \beta 42$ and tau (total [T-tau], and phosphorylated [Ptau]). Reduced CSF A $\beta 42$ and increased P-tau are believed to reflect $A \beta$ and tau pathologies in AD, while increased T-tau is a more non-specific marker of neuronal injury (Blennow et al. 2010). PET imaging can be used to directly visualize aggregates of both $\mathrm{A} \beta$ and tau in the brain. However, both CSF measurements and PET imaging have significant hurdles. They are invasive requiring a lumbar puncture (LP) or the injection of a radioactive ligand, are time-consuming for the clinician or cumbersome for the tested individual and LP may have side-effects (e.g. post-spinal headache is the most common). They are also relatively expensive, and the availability is limited especially in primary care. There is therefore a great need for less invasive, cost-effective and easily accessible biomarkers, preferably blood tests.

Several assays have recently been developed to measure blood-based tau phosphorylated at threonine (Thr) 181 (P-tau181). Studies using these assays found increased plasma levels of Ptau181 in patients with AD dementia or Down's syndrome compared to cognitively unimpaired individuals, and also found correlations between plasma P-tau181 and higher levels of A $\beta$ PET and tau PET uptake (Tatebe et al. 2017, Mielke et al. 2018, Yang et al. 2018). Together, these results are promising for the use of plasma P-tau181 as an AD biomarker, but several issues related to the performance of plasma P-tau181 are still unclear. For example, it is not known if plasma P-tau181 is a useful biomarker to i) differentiate $\mathrm{AD}$ from non-AD neurodegenerative diseases, ii) to predict future progression to AD dementia in non-demented people or iii) to identify individuals with pathological levels of Tau PET uptake. It is also unclear how plasma levels of P-tau181 correlate with CSF P-tau181 levels, or how the diagnostic performance compares with other recently described plasma biomarkers in AD (Chouraki et al. 2015, Mattsson et al. 2016, Mattsson et al. 2019, Pase et al. 2019). We therefore designed a study to test the relationship between plasma Ptau181 and CSF P-tau181, as well as PET imaging of tau and A $\beta$ aggregates. Further, we investigated whether plasma P-tau181 could differentiate between AD dementia and non-AD neurodegenerative diseases and predict progression to AD dementia over time in non-demented individuals. The predictive value of plasma P-tau181 was also compared to other relevant plasma biomarkers (i.e., T-tau, A $\beta 42 / \mathrm{A} \beta 40$ ratio and neurofilament light [NfL]).

\section{METHODS}

The study was approved by the Regional Ethics Committee in Lund, Sweden, and all participants gave their informed consent to participate in the study. Approval for PET imaging was obtained from the Swedish Medical Products Agency and the local Radiation Safety Committee at Skåne University Hospital.

\section{Participants}

This project was done as part of the Swedish BioFINDER study and included two separate cohorts recruited at Skåne University Hospital and the Hospital of Ängelholm, Sweden. Cohort 1 included participants $(n=182)$ enrolled between November 2014 and January 2018 who underwent $\left[{ }^{18}\right.$ F]flortaucipir PET imaging, and cohort 2 included non-demented participants $(n=344)$ enrolled between January 2010 and December 2014 who were followed longitudinally. The demographics of each cohort are described in Table 1 . The inclusion criteria for cognitively healthy elderly were 1) absence of cognitive symptoms as assessed by a physician with special interest in cognitive disorders, 2) age $\geq 60$ years, 3) MMSE 28-30 points at screening visit, 4) did not fulfill the criteria for mild cognitive impairment (MCI) or any dementia disorder, and 5) fluency in Swedish. The 
exclusion criteria were 1) significant unstable systemic illness or organ failure, such as terminal cancer, that made it difficult to participate in the study, 2) current significant alcohol or substance misuse and 3) significant neurological or psychiatric illness. The inclusion criteria for patients with subjective cognitive decline (SCD) or MCI (defined using criteria by (Petersen 2004)) were (1) referred to a participating memory clinic because of cognitive complaints, (2) age 60 to 80 years, (3) did not fulfill the criteria for any dementia disorder and (4) fluency in Swedish. The exclusion criteria were 1) significant unstable systemic illness or organ failure, such as terminal cancer, that made it difficult to participate in the study, 2) current significant alcohol or substance misuse and 3) cognitive impairment that without doubt could be explained by other specific nonneurodegenerative disorders, such as brain tumor or subdural hematoma. Following neuropsychological assessment including a test battery evaluating verbal ability, episodic memory function, visuospatial construction ability, and attention and executive functions, patients were classified as SCD or MCI as previously described(Mattsson et al. 2016). In accordance with the research framework by the National Institute on Aging-Alzheimer's Association(Jack et al. 2018), study participants with SCD were analyzed together with the cognitively healthy participants (and combined in the cognitively unimpaired group).

In cohort 2, the study participants (i.e., participants who were cognitively unimpaired or had MCI at baseline) were followed over time with repeated cognitive, neurologic and psychiatric assessments by a physician, and also underwent repeated cognitive testing, MRI, and collection of CSF and blood. The follow-up visits were performed every two years in the cognitively healthy group and every year for SCD and MCI patients (mean follow-up 4.9 years, SD=1.3 years).

In cohort 1 , the study protocol was a priori designed to enroll cognitively unimpaired participants with a high prevalence $(>50 \%)$ of $\mathrm{A} \beta$ positivity $(\mathrm{A} \beta+)$, and only $\mathrm{A} \beta+\mathrm{MCI}$ patients, because the focus was to study tau pathology in the pre-dementia stages of AD. In cohort 1, we also included patients with AD dementia, who fulfilled the DSM-5 criteria for major neurocognitive disorder (dementia) due to AD. All those were A $\beta+$ (Jack et al. 2018). The non-AD neurodegenerative diseases group included patients with Parkinson's disease with dementia (PDD), Dementia with Lewy Bodies (DLB), Frontotemporal dementia (FTD) (all fulfilling the DSM-5 criteria for the respective disease), Parkinson's disease (PD) (fulfilling the criteria defined by Gelb et al.(Gelb et al. 1999)), Progressive Supranuclear Palsy (PSP) (fulfilling the criteria defined by Litvan et al. and Höglinger et al.(Litvan et al. 1996, Hoglinger et al. 2017) and corticobasal syndrome (CBS) (fulfilling the criteria defined by Armstrong et al.(Armstrong et al. 2013)).

\section{Plasma and CSF sampling and analysis}

Blood and CSF samples were collected in the morning during the same visit with participants nonfasting. For each study participant, blood was collected in 6 EDTA-plasma tubes (Vacutainer® $\mathrm{K}_{2}$ EDTA tube, BD Diagnostics) and centrifuged $\left(2000 \mathrm{~g},+4^{\circ} \mathrm{C}\right)$ for $10 \mathrm{~min}$. Following centrifugation, plasma from all 6 tubes was transferred into one $50 \mathrm{ml}$ polypropylene tubes tube, mixed and $1 \mathrm{ml}$ was aliquoted into $1.5 \mathrm{ml}$ polypropylene tubes and stored at $-80^{\circ} \mathrm{C}$ within $30-60 \mathrm{~min}$ of collection. Lumbar puncture and CSF handling followed a structured protocol (Palmqvist et al. 2014). The laboratory technicians performing the biochemical analyses were blinded to the clinical data. CSF from both cohorts was analyzed for A $\beta 42$, A $\beta 40$ and T-tau using ELISA (Euroimmun AG, Lübeck, Germany) according to manufacturer's recommendations. CSF P-tau181 was quantified using ELISA, as described in eMethods.

Plasma P-tau181 was analyzed in both cohorts as previously described(Mielke et al. 2018). In short, the assay was performed on a streptavidin plate on the Meso Scale Discovery (MSD) platform. Biotinylated-AT270 was used as capture antibody (anti-pT181 Tau antibody; Thermo Fisher, catalog number: MN1050) and SULFO-TAG-LRL (anti-tau monoclonal antibodies developed by Lilly Research Laboratory) as detector. Antibodies were conjugated with Sulfo-NHSBiotin (Thermo Scientific, catalog number: 21327) or MSD GOLD SULFO-TAG NHS-Ester 
(MSD, catalog number: R91AO) according to the manufacturer's protocol. The assay was calibrated using a recombinant tau (4R2N, NCBI tau v2) protein that was phosphorylated in vitro. In cohort 1, five (2.7\%) samples from non-AD neurodegenerative diseases were below the level of detection (LOD, $0.54 \mathrm{pg} / \mathrm{ml}$ ). In cohort 2, $15(4.4 \%)$ samples were below the level of detection, including nine (2.6\%) A $\beta$ - cognitively unimpaired, four (1.2\%) A $\beta$ - MCI and two A $\beta+$ cognitively unimpaired $(0.6 \%)$. All plasma samples with P-tau181 concentrations below the LOD were included in statistical analysis using values extrapolated from the standard curve. Outliers with plasma P-tau181 levels of more than 3 SD above the mean $(n=7,1.3 \%$ of all values) were excluded from statistical analysis.

In cohort 2, plasma $A \beta 42, A \beta 40$ and T-tau were measured using Elecsys fully-automated immunoassays on a cobas e 601 analyzer as described previously (Palmqvist et al. 2019), and plasma NFL concentration was measured using the monoclonal antibodies as in the NF-light assay (UmanDiagnostics AB, Sweden) together with an in-house calibrator (purified bovine NfL) transferred onto the Simoa platform using a homebrew kit (Quanterix, Lexington, MA, USA), as described previously (Hansson et al, 2017).

\section{Tau and A $\beta$ PET imaging and processing}

$\left[{ }^{18} \mathrm{~F}\right]$ flortaucipir and $\left[{ }^{18} \mathrm{~F}\right]$ flutemetamol were synthesized at Skåne University Hospital, Lund, and PET scans were performed on a GE Discovery 690 PET scanner (Flortaucipir; General Electric Medical Systems, Chicago, IL) and a Philips Gemini TF 16 scanner (Flutemetamol; Philips Healthcare, Amsterdam, the Netherlands), respectively, as described previously (Hahn et al. 2017, Ossenkoppele et al. 2019).

$\left[{ }^{18} \mathrm{~F}\right]$ flortaucipir PET was performed in cohort 1 . The mean injected dose of $\left[{ }^{18} \mathrm{~F}\right]$ flortaucipir was $\approx 370 \mathrm{MBq}$, and participants underwent a PET scan 80-100 minutes after injection. Images were motion corrected with the AFNI 3dvolreg, time averaged, and rigidly coregistered to the skullstripped MRI scan. Standardized uptake value ratio (SUVR) images were created using inferior cerebellar gray matter as reference region (Maass et al. 2017). FreeSurfer (version 5.3) parcellation of the T1-weighted MRI scan was applied to the PET data transformed to participants' native T1 space to extract mean regional SUVR values for each participant in four predefined ROI including inferior temporal cortex and three regions corresponding to different image-based stages of tau as described in Cho et al.(Cho et al. 2016): the Braak I/II (entorhinal cortex), III/IV (parahippocampal gyrus, fusiform gyrus, amygdala, inferior temporal and middle temporal gyri) and V/VI (posterior cingulate gyrus, caudal anterior cingulate gyrus, rostral anterior cingulate gyrus, precuneus, inferior parietal lobule, superior parietal lobule, insula, supramarginal gyrus, lingual gyrus, superior temporal gyrus, medial orbitofrontal gyrus, rostral middle frontal gyrus, lateral orbitofrontal gyrus, caudal middle frontal gyrus, superior frontal gyrus, lateral occipital gyrus, precentral gyrus, postcentral gyrus and paracentral gyrus) ROIs. For voxel-wise analysis between $\left[{ }^{18} \mathrm{~F}\right]$ flortaucipir and CSF and plasma P-tau181 the MR and PET images were transformed into Montreal Neurological Institute space (2mm MNI152 MRI template) and voxel-wise correlations were made using multiple regressions adjusting for age in SPM12 (http://www.fil.ion.ucl.ac.uk/spm). Images were thresholded using family-wise error (FWE) correction at $\mathrm{p}<0.01$. The thresholded images were overlaid on a Population-Average, Landmark- and Surface-based (PALS) image (Van Essen 2005) using CARET v5.65 (Van Essen Lab; http://brainvis.wustl.edu). For some analyses,

$\left[{ }^{18}\right.$ F]flortaucipir data were binarized based on the SUVR cutoff of 1.3 (Ossenkoppele et al. 2018).

$\left[{ }^{18} \mathrm{~F}\right]$ flutemetamol PET was done in both cohort 1 and cohort 2 , and the mean injected dose was $\approx 185 \mathrm{MBq}$. PET images were acquired between 90 and 110 minutes after injection. The scanning and processing procedures have been described previously(Palmqvist et al. 2014, Palmqvist et al. 2017). The weighted mean standardized uptake value ratio (SUVR) from a global neocortical region of interest was calculated relative to a composite reference region (white matter, cerebellum and brainstem)(Palmqvist et al. 2017, Palmqvist et al. 2019). 


\begin{abstract}
A $\beta$ positivity
Cutoffs for CSF A $\beta 42(<510 \mathrm{pg} / \mathrm{ml})$ and $\left[{ }^{18} \mathrm{~F}\right]$ flutemetamol PET SUVR $(>0.743)$ were computed using Gaussian mixture modelling (ref Mattsson, Leuzy, under review). Study participants who underwent both lumbar puncture and $\mathrm{A} \beta$ PET imaging were considered $\mathrm{A} \beta$ positive if either CSF $\mathrm{A} \beta 42$ or $\mathrm{A} \beta$ PET measures were abnormal.
\end{abstract}

\title{
Statistical analysis
}

Differences in baseline characteristics were analyzed using one-way analysis of variance (ANOVA) and chi-square tests. Correlations between plasma P-tau181 and CSF P-tau181 and between plasma P-tau181 and $\left[{ }^{18} \mathrm{~F}\right]$ flortaucipir were examined with Spearman test. Group differences in the biomarker levels were assessed using univariate general linear models (GLM) adjusting for age and sex as covariates and least significant difference (post hoc) tests for pairwise group comparisons.

Cutpoints were defined by Youden index (maximizing the sum of sensitivity and specificity). Diagnostic accuracies of biomarkers were assessed with receiver operating characteristic (ROC) curve analysis and logistic regression models. We used a bootstrap procedure ( $n=2000$ bootstrap samples) to compare areas under two ROC curves and compute $95 \%$ confidence intervals $(\mathrm{CI})$ of the sensitivities and specificities of the optimal thresholds.

To assess associations between plasma P-tau181 (used as a continuous variable or by tertiles) and risk of incident AD dementia we used Cox proportional hazard regression models adjusting for age, sex and years of education. In a secondary analysis, we additionally included other potential plasma biomarkers of AD (i.e. t-tau A $\beta 42 / A \beta 40$ and NfL). Log-transformed biomarker data were standardized within the sample and all study participants were censored at their last follow-up visit or diagnosis of AD dementia. Results were expressed as hazard ratio (HR) with $95 \%$ CI. The proportionality of hazards assumption was assessed using the Schoenfeld residuals.

Associations between log-transformed plasma P-tau181 and continuous A $\beta$ PET uptake were tested with non-linear polynomial spline models (using I-spline basis), to detect A $\beta$ PET thresholds for increased P-tau181 (defined as the A $\beta$ level where the spline increased at least two standard errors from baseline).

The Akaike Information Criterion (AIC), a model selection tool, was used to compare models with the same outcome and individuals (a decrease in AIC $<2$ indicates a significantly better fit for the model with smaller AIC). Log-transformed plasma P-tau181 values were used in all regression analyses. Two-sided $\mathrm{p}<0.05$ were considered statistically significant. All analyses were performed using SPSS version 24 (IBM, Armonk, NY, US) and R version 3.5.3 (including the packages $p R O C$, survival, survminer and splines 2 ).

\section{RESULTS}

\section{Cohort 1}

\section{Participants}

Cohort 1 included 64 cognitively unimpaired participants (38 [60\%] A $\beta+; 54$ were cognitively healthy individuals and 10 were participants with SCD), $28 \mathrm{~A} \beta+\mathrm{MCI}, 38 \mathrm{~A} \beta+\mathrm{AD}$ dementia patients, and 52 patients with non-AD neurodegenerative diseases (26 [52\%] A $\beta+;$ PD [n=11], PDD [n=17], PSP [n=6], DLB [n=6], CBS [n=6], SD [n=2] and bvFDT [n=4]). Rates of A $\beta$ positivity in non-AD neurodegenerative diseases $(52 \%)$ were consistent with previously published data(Ossenkoppele et al. 2015). Baseline characteristic of cohort 1 are provided in Table 1. Years of education and sex did not differ between the groups, while there were significant differences in age $(\mathrm{F}=3.6, \mathrm{p}=0.014)$, MMSE $(\mathrm{F}=47.8, \mathrm{p}<0.001)$ and $\mathrm{CSF}$ AD biomarkers $(\mathrm{A} \beta 42 \mathrm{~F}=18.3, \mathrm{p}<0.001$; $\mathrm{T}$ tau $\mathrm{F}=35.8, \mathrm{p}<0.001 ; \mathrm{P}-\operatorname{tau} 181 \mathrm{~F}=52.3, \mathrm{p}<0.001)$. The mean age in the non-AD neurodegenerative diseases group was lower than in the cognitively unimpaired group (pairwise post hoc test: 
$\mathrm{p}=0.001$ ). MMSE scores were higher in the cognitively unimpaired group and lower in $\mathrm{A} \beta+\mathrm{AD}$ dementia patients compared with other groups (pairwise post hoc test: all $p<0.001$ ).

\section{Correlations between plasma and CSF P-tau181}

In cohort 1 , we found a strong positive correlation between plasma and CSF levels of P-tau181 $(\mathrm{Rs}=0.72, \mathrm{p}<0.001, \mathrm{n}=172)$. When stratifying by $\mathrm{A} \beta$ status, the correlation was significant in $\mathrm{A} \beta+$ $(\mathrm{Rs}=0.68, \mathrm{p}<0.001, \mathrm{n}=125)$ but not in $\mathrm{A} \beta$ - individuals $(\mathrm{Rs}=0.11, \mathrm{p}=0.45, \mathrm{n}=47)$ (Figure $1 \mathrm{~A})$.

\section{Associations with ${ }^{18}$ F $]$ flortaucipir}

$\left[{ }^{18} \mathrm{~F}\right]$ flortaucipir PET was performed in 174 study participants. We first studied associations between plasma $\mathrm{P}$-tau 181 and $\left[{ }^{18} \mathrm{~F}\right]$ flortaucipir retention in commonly used $a$ priori defined brain regions linked to tau pathology in AD, including the Braak I/IV ROI (temporal "meta-ROI") as well as the Braak I/II, III/IV, V/VI ROIs and inferior temporal cortex(Ossenkoppele et al. 2019). Higher concentrations of P-tau181 were associated with increased $\left[{ }^{18} \mathrm{~F}\right]$ flortaucipir retention in the temporal meta-ROI (Braak I-IV ROI) in the whole cohort $(\mathrm{Rs}=0.64, \mathrm{p}<0.001)$ and in $\mathrm{A} \beta+(\mathrm{Rs}=0.69, \mathrm{p}<0.001)$ but not in $A \beta-(R s=0.12, p=0.43)$ individuals (Figure $1 \mathrm{~B}$ and eTable 1). The results were similar for the other four ROIs (eFigure 1). In the cognitively unimpaired group, plasma levels of P-tau181 were positively related to $\left[{ }^{18} \mathrm{~F}\right]$ flortaucipir retention in the earliest tau region (the Braak I/II ROI, $\mathrm{Rs}=0.29, \mathrm{p}=0.02$ ) but not in other regions, whereas in the cognitively impaired participants (A $\beta+$ $\mathrm{MCI}$ and $\mathrm{A} \beta+\mathrm{AD}$ dementia) the correlations were significant in all regions (Rsrange 0.49-0.60, all $\mathrm{p}<0.001$ ) except the early Braak I/II ROI (eTable 1). Voxel-wise analyses revealed that plasma Ptau181 was mainly associated with $\left[{ }^{18} \mathrm{~F}\right]$ flortaucipir PET uptake in temporo-parietal regions (Fig1 C).

Next, we studied whether the plasma P-tau181 values varied between individuals at different stages of tau pathology as defined with $\left[{ }^{18} \mathrm{~F}\right]$ flortaucipir PET. The cohort was divided into those with normal $\left[{ }^{18} \mathrm{~F}\right]$ flortaucipir retention in all Braak ROIs (Tau PET negative), and those with abnormal retention in either i) Braak I/II (but not III-VI), ii) Braak III/IV (but not V-VI) or iii) Braak V/VI. We observed that P-tau181 concentrations differed between the Braak I/II+, III/IV+ and V/VI+ groups compared to those with normal $\left[{ }^{18} \mathrm{~F}\right]$ flortaucipir retention $(\mathrm{F}=43.5, \mathrm{p}<0.001)$. Plasma P-tau181 were increased in the Braak III/IV+ and V/VI+ compared with Tau PET-negative (pairwise post hoc test: both $\mathrm{p}<0.001$; Figure $2 \mathrm{~A}$ ). There was also a trend for higher P-tau181 levels in the Braak I/II+ group (pairwise post hoc test: $\mathrm{p}=0.056$ ).

To establish whether plasma P-tau181 was associated with normal vs abnormal Tau PET scans we binarized $\left[{ }^{18} \mathrm{~F}\right]$ flortaucipir data. In ROC curve analysis, P-tau 181 accurately predicted abnormal Tau PET status in temporal meta-ROI (AUC 0.87 [95\%CI 0.81\%-0.92\%], sensitivity 80\% [95\%CI 70\%-90\%], specificity 78\% [95\%CI 70\%-85\%]; Figure 2B and eTable 2) and the other four predefined ROIs (AUC range 0.81-0.91, Figure 2B and eTable 2).

\section{Plasma P-tau181 in AD and non-AD neurodegenerative diseases}

$\mathrm{P}$-tau181 concentrations varied between the diagnostic groups $(\mathrm{F}=36.0, \mathrm{p}<0.001$; Figure $3 \mathrm{~A})$. We found higher levels of P-tau181 in $A \beta+$ cognitively unimpaired, $A \beta+M C I$ and $A \beta+A D$ dementia than in $A \beta$ - cognitively unimpaired (pairwise post hoc test: $p=0.04, p<0.001$ and $p<0.001$, respectively) and non-AD neurodegenerative diseases (pairwise post hoc test: all $\mathrm{p} \leq 0.001$ ). $\mathrm{P}$ tau181 was also increased in $\mathrm{A} \beta+\mathrm{MCI}$ and $\mathrm{A} \beta+\mathrm{AD}$ dementia compared with $\mathrm{A} \beta+$ cognitively unimpaired (pairwise post hoc test: both $\mathrm{p}<0.001$ ).

Further, plasma P-tau181 accurately discriminated AD dementia $(n=38)$ from non-AD neurodegenerative diseases $(n=52)$ with AUC of 0.93 (95\% CI 0.88-0.98). When using the Youden index derived cutoff, the sensitivity and specificity were $92 \%$ (95\% CI 82\%-100\%) and 87\% (95\%CI 77\%-94\%), respectively (eTable 3). We next compared the diagnostic performance of plasma P-tau181 with CSF P-tau181 and $\left[{ }^{18} \mathrm{~F}\right]$ flortaucipir SUVR in the temporal meta-ROI (Braak I-IV) in a subcohort of 83 patients (35 AD dementia and 48 non-AD neurodegenerative disorders [10 PD, 16 PDD, 5 PSP, 5 DLB, 6 CBS, 2 SD and 4 bvFTD]) where all these measure were 
available. The AUC, sensitivity and specificity were slightly lower for plasma P-tau181 (Figure 3 and eTable 3), but the differences were small (AUC 0.02-0.03, sensitivity 3\%-6\% and specificity $2 \%-6 \%)$ and not statistically significantly different from each other ( $\mathrm{p}=0.28$ for AUC plasma Ptau181 vs CSF P-tau181, and $\mathrm{p}=0.10$ for AUC plasma P-tau181 vs $\left[{ }^{18} \mathrm{~F}\right]$ flortaucipir SUVR).

\section{Cohort 2}

\section{Participants}

Cohort 2 included 219 cognitively unimpaired participants and 125 MCI patients (Table 1) of whom 93 cognitively unimpaired participants (42\%) and 81 MCI patients $(65 \%)$ were A $\beta+$. The prevalences of $A \beta$ positivity were consistent with previously published data(Ossenkoppele et al. 2015). Baseline characteristic of cohort 2 are provided in Table 1.

Age $(\mathrm{F}=5.9, \mathrm{p}=0.001)$, sex $\left(\chi^{2}=16.1, \mathrm{p}=0.001\right)$, years of education $(\mathrm{F}=3.7, \mathrm{p}=0.012)$, MMSE $(\mathrm{F}=31.6, \mathrm{p}<0.001)$ and $\mathrm{CSF} A D$ biomarkers $(\mathrm{A} \beta 42 \mathrm{~F}=186.4, \mathrm{p}<0.001$; $\mathrm{T}$-tau $\mathrm{F}=27.7, \mathrm{p}<0.001$; $\mathrm{P}$ tau181 $\mathrm{F}=46.6, \mathrm{p}<0.001)$ differed by diagnostic group. The $\mathrm{A} \beta$ - MCI group was younger than all the other groups (pairwise post hoc test: all $p<0.002$ ). There were more men in the A $\beta$ - MCI group compared with the $A \beta$ - cognitively unimpaired and $A \beta+$ cognitively unimpaired groups (both $\mathrm{p}=0.01$ ) and in the $\mathrm{A} \beta+\mathrm{MCI}$ patients compared with the $\mathrm{A} \beta$ - cognitively unimpaired group $(\mathrm{p}<0.001)$. Education was on average one year shorter in the A $\beta$ - MCI and A $\beta+$ MCI groups compared with the cognitively unimpaired and $A \beta+$ cognitively unimpaired groups (pairwise post hoc test: all $\mathrm{p}=0.02$ ). MMSE scores were lower in the MCI groups than in the cognitively unimpaired groups (pairwise post hoc test: all $\mathrm{p}<0.001$ ) and in $\mathrm{A} \beta$ - MCI compared with $\mathrm{A} \beta+\mathrm{MCI}$ (pairwise post hoc test: $\mathrm{p}=0.023$ ).

Similar to cohort 1 , we found significant positive correlation between plasma P-tau181 and CSF P-tau181 (Rs=0.48, $\mathrm{p}<0.001, \mathrm{n}=343)$ and also that the correlations were significant in $\mathrm{A} \beta+$ $(\mathrm{Rs}=0.55, \mathrm{p}<0.001, \mathrm{n}=173)$ but not in $\mathrm{A} \beta-(\mathrm{Rs}=0.11, \mathrm{p}=0.15, \mathrm{n}=170)$ individuals (eFigure 2$)$.

\section{Plasma P-tau181 in diagnostic groups}

P-tau181 levels varied between different diagnostic groups $(\mathrm{F}=39.5, \mathrm{p}<0.001$; Figure 4B $)$. We found higher concentrations of $\mathrm{P}$-tau 181 in $\mathrm{A} \beta+$ cognitively unimpaired and $\mathrm{A} \beta+\mathrm{MCI}$ patients compared with $A \beta$ - cognitively unimpaired and $A \beta$ - MCI patients and also in $A \beta+$ MCI patients compared with $A \beta+$ cognitively unimpaired (pairwise post hoc test: all $p \leq 0.001$; Figure $4 B$ ).

\section{Plasma P-tau181 vs $\left[{ }^{18}\right.$ F $]$ flutemetamol PET}

$\left[{ }^{18} \mathrm{~F}\right]$ flutemetamol PET was performed in 318 study participants. Increased levels of P-tau181 correlated with increased $\left[{ }^{18} \mathrm{~F}\right]$ flutemetamol SUVR in the whole cohort $(\mathrm{Rs}=0.44, \mathrm{p}<0.001)$ and in $\mathrm{A} \beta+$ individuals $(\mathrm{Rs}=0.43, \mathrm{p}<0.001, \mathrm{n}=161)$. The correlation coefficients were higher for plasma $\mathrm{P}$ tau181 than plasma $A \beta 42$ and comparable for P-tau181 and plasma A $\beta 42 / A \beta 40$ (eTable 4). Ptau181 accurately differentiated individuals with normal versus pathological $\left[{ }^{18} \mathrm{~F}\right]$ flutemetamol status when including all study participants (AUC 0.81 [95\% CI $0.76 \%-0.86 \%$ ], and when used combined with plasma $\mathrm{A} \beta 42 / \mathrm{A} \beta 40$ ratio $(\mathrm{AUC}=0.84,95 \% \mathrm{CI}=0.80-0.89$ ). The results were similar in cognitively unimpaired participants and MCI patients (eTable 5).

Using non-linear spline models with continuous $\left[{ }^{18} \mathrm{~F}\right]$ flutemetamol load as predictor of plasma P-tau181, we determined the $\mathrm{A} \beta$ thresholds for when P-tau181 levels increased (Figure 4A). Plasma P-tau 181 increased significantly at $\left[{ }^{18} \mathrm{~F}\right]$ flutemetamol 0.70 SUVR. This was slightly after similar thresholds for CSF P-tau181 (SUVR 0.63) and close to a threshold for $\left[{ }^{18} \mathrm{~F}\right]$ flutemetamol positivity (SUVR 0.743 ref: Mattsson, Leuzy, under review). These results show that increases in plasma $\mathrm{P}$-tau181 start early in $\mathrm{AD}$, just before the time of $\mathrm{A} \beta$ positivity, and they were replicated in cohort 1 (eFigure 1). 
Among 344 participants in cohort 2, 332 underwent follow-up clinical examinations. Over a mean follow-up period of 4.9 years ( $\mathrm{SD}=1.3$ years), 62 (18.7\%) individuals developed AD dementia and $33(9.9 \%)$ other neurodegenerative disorders (Vascular dementia [ $n=15]$, DLB [n=6], bvFTD [ $n=2]$, PD [n=4], normal-pressure hydrocephalus [n=2], CADASIL [ $n=1]$, dementia unspecified [n=3]). Cox proportional hazard regression models adjusted for age, sex and education showed that each 1 SD increment in the log of plasma P-tau 181 was associated with $280 \%$ greater risk of AD dementia $(\mathrm{HR}=3.8,95 \% \mathrm{CI}=2.8-5.2, \mathrm{p}<0.001)$. Compared to the lowest tertile, both the highest and the middle tertiles of plasma P-tau181 were associated with increased risk of incident AD dementia $(\mathrm{HR}=27.2$, 95\% CI=6.6-112.5, $\mathrm{p}<0.001$ and $\mathrm{HR}=5.8,95 \% \mathrm{CI}=1.3-25.7, \mathrm{p}=0.02$, respectively; Figure 4C). We found similar associations between plasma P-tau181 (continuous) and increased risk of dementia $(\mathrm{HR}=3.6,95 \% \mathrm{CI}=2.6-5.0, \mathrm{p}<0.001, \mathrm{n}=325)$ after additional adjustment for plasma $\mathrm{T}$-tau, $\mathrm{A} \beta 42 / \mathrm{A} \beta 40$ and NfL. Furthermore, none of the latter biomarkers were independently related to the risk of $\mathrm{AD}$ dementia in the multivariate analyses including all plasma biomarkers ( $\mathrm{T}$-tau $\mathrm{HR}=0.98$, 95\% CI=0.73-1.32; $\mathrm{A} \beta 42 / \mathrm{A} \beta 40 \mathrm{HR}=0.81,95 \% \mathrm{CI}=0.64-1.0 ; \mathrm{NfL} \mathrm{HR}=1.1,95 \% \mathrm{CI}=0.79-1.44)$. In a secondary analysis stratified by baseline diagnosis, plasma P-tau181 was associated with increased risk of $\mathrm{AD}$ dementia in the cognitively unimpaired participants $(\mathrm{HR}=2.5,95 \% \mathrm{CI}=1.2-5.1, \mathrm{p}=0.01)$ and MCI patients $(\mathrm{HR}=3.1,95 \% \mathrm{CI}=2.1-4.4, \mathrm{p}<0.001)$, and after additional adjustment for plasma $\mathrm{T}$-tau, $\mathrm{A} \beta 42 / \mathrm{A} \beta 40$ and $\mathrm{NfL}$ (cognitively unimpaired $\mathrm{HR}=2.4,95 \% \mathrm{CI}=1.0-5.5, \mathrm{p}=0.04$; $\mathrm{MCI}$ $\mathrm{HR}=3.0,95 \% \mathrm{CI}=2.0-4.5, \mathrm{p}<0.001)$.

\section{DISCUSSION}

The main findings of this study were that plasma P-tau181 1) correlated to CSF P-tau181, 2) identified increased Tau PET ligand uptake, 3 ) increased along the AD continuum, from A $\beta$ cognitively unimpaired participants, $\mathrm{A} \beta+$ cognitively unimpaired, $\mathrm{MCI}$ and $\mathrm{AD}$ dementia, 4) accurately distinguished between AD and non-AD neurodegenerative diseases with high accuracy, and 5) predicted longitudinal conversion to $\mathrm{AD}$ dementia with greater precision than previously established plasma AD biomarkers, including A $\beta 42 / \mathrm{A} \beta 40$ ratio, NfL and T-tau. Taken together, these results show that plasma P-tau181 is related to several key aspects of AD pathophysiology and support the use of plasma P-tau181 for screening, clinical diagnosis, differential diagnosis, and prognosis with a precision previously only seen in CSF and PET biomarkers. Notably, plasma Ptau181 concentration showed larger fold change and less overlap between $A \beta$-positive and negative subject than plasma $A \beta 42 / A \beta 40$ ratio (refs: Ovod + Nakamura + Janelidze), and could thus potentially serve as an A $\beta$ pathology "sensor" in the blood.

The correlations with CSF P-tau181 found in both cohorts resemble the association between plasma P-tau181 and CSF P-tau181 in a much smaller study by Tatebe et al. (N=11)(Tatebe et al. 2017) and suggests that plasma P-tau 181 to a large degree is derived from the central nervous system. A novel finding in our study was that plasma P-tau181 and CSF P-tau181 only correlated in $\mathrm{A} \beta+$ individuals. This was likely due to greater dynamic ranges of both plasma and CSF P-tau181 in $A \beta$ positive subjects, making it easier to detect significant correlations. It is possible that CSF and blood P-tau181 are differently regulated with different pathophysiological secretion depending on A $\beta$ status. Supporting this, plasma P-tau181 had greater variability than CSF P-tau181 in A $\beta$ individuals, and some individuals had considerable increases in plasma P-tau181 without increases in CSF P-tau181 (Figure 1 and eFigure 2). Other studies have shown much weaker correlations between plasma T-tau and CSF T-tau levels than the correlations described here for Ptau181(Zetterberg et al. 2013, Mattsson et al. 2016, Palmqvist et al. 2019, Pase et al. 2019), which may be due to tau expression in peripheral tissues. Therefore, the stronger correlations between plasma and CSF levels of P-tau181 compared to total-tau likely reflects that under normal conditions, and in chronic neurodegenerative disorders, P-tau181 is more brain-specific (and more specific for AD), while T-tau can potentially also be produced outside the central nervous system (Couchie et al. 1992) (and is also increased due to other brain conditions than AD). The present findings indicate that plasma P-tau181 is superior to previously published results for plasma T-tau, 
both for diagnostic purposes and to detect pathological brain changes in AD (Mattsson et al. 2016, Mielke et al. 2018). Importantly, we found that plasma P-tau181, but not T-tau, was independently associated with increased risk of progression to AD dementia. (eTable 4).

The correlations between P-tau181 and Tau PET indicate that the increase in plasma Ptau181 is related to the build-up of tau deposits in AD, since Tau PET is closely correlated to brain tau deposits(Smith et al. 2016, Smith et al. 2018). Plasma P-tau181 correlated to Tau PET in all tested regions in the overall cohort 1 , and to all regions except the Braak I/II region (entorhinal cortex) in $A \beta+$ cognitively impaired individuals. A few factors may explain the lack of correlation with Tau PET in the entorhinal cortex in cognitively impaired individuals. Entorhinal cortex is one of the earliest sites engaged by tau pathology in AD and one of the first sites with increased Tau PET signal(Scholl et al. 2018) and thus it is possible that Tau PET in this region may be saturated in this brain region in most symptomatic individuals. In contrast, the only correlations to Tau PET in cognitively unimpaired individuals were seen in the entorhinal cortex, supporting the use of plasma P-tau181 as a very sensitive test for tau pathology. Notably, the only previous study correlating plasma P-tau181 with Tau PET in entorhinal cortex similarly did not find correlations in A $\beta+A D$ dementia, but only in A $\beta+$ cognitively unimpaired and MCI, in line with our findings(Mielke et al. 2018).

Besides correlating to continuous regional Tau PET, plasma P-tau181 also identified individuals with abnormal Tau PET ligand retention in the Braak composites (AUC 0.81-0.91). Plasma P-tau181 may therefore be useful as a non-invasive biomarker to predict a positive Tau PET scan, for example as a screening tool for enrichment in clinical trials aiming to enroll tau positive subjects. We also applied a staging system, where individuals were grouped based on regional Tau PET signals, into Tau PET-, Braak I/II+, III/IV+ and V/VI+ stages. Using this system, plasma Ptau181 levels increased numerically stepwise between the stages. There were no significant differences between the positive stages, which may partly be a power issue, since the positive groups were rather small ( $\mathrm{N}=12$ to 33$)$. Future studies may characterize how plasma P-tau181, together with other measures, can be fine-tuned to predict different classes of Tau PET positivity.

Tau PET is strongly correlated to level of cognitive impairment in $\mathrm{AD}$ (Scholl et al. 2016, Ossenkoppele et al. 2018). Given the correlations between plasma P-tau181 and Tau PET, the findings in our cohorts of stepwise increases in plasma P-tau181 from A $\beta$ - cognitively unimpaired to $A \beta+$ cognitively unimpaired and further to $A \beta+\mathrm{MCI}$ (Figures 3 and 4 ) were therefore expected. The lack of significant difference between $\mathrm{A} \beta+\mathrm{MCI}$ and $\mathrm{AD}$ dementia indicates that plasma $\mathrm{P}$ tau181 eventually reaches a plateau. These results are largely similar to data from previous studies by Mielke et al. (Tatebe et al. 2017, Mielke et al. 2018), except that our data suggest that plasma Ptau181 may be increased already in preclinical stages of AD. This is similar to what has been described previously for CSF P-tau181(Mattsson et al. 2017, Mattsson et al. 2018).

Differential diagnosis of AD dementia from other diseases is difficult on clinical grounds, as reflected by the modest correlation between clinical AD diagnosis and neuropathological confirmation of the disease(Beach et al. 2012). Tau PET has excellent performance to differentiate $\mathrm{AD}$ dementia from non-AD neurodegenerative diseases, since abnormal increase in Tau PET is very rare in most other dementias(Ossenkoppele et al. 2018). We show that plasma P-tau181 has very similar performance (AUC 0.95) as Tau PET (AUC 0.98) when differentiating AD from other neurodegenerative disorders, indicating that plasma P-tau181 has a potential as an alternative to Tau PET for differential diagnosis between $\mathrm{AD}$ (at the dementia stage) and other neurodegenerative diseases.

The prediction of future longitudinal impairment and dementia is a key question for clinical management of individuals with mild cognitive deficits, and for design of clinical trials in nondemented stages of AD. It is well known that CSF biomarkers, including P-tau181, are altered several years prior to dementia, and predict future conversion to AD dementia(Hansson et al. 2006), but blood tests would clearly be preferable to CSF. In the present study, we show that P-tau181 accurately predicts conversion to AD dementia in non-demented people. In models combining plasma P-tau181 and other potential plasma AD biomarkers, including T-tau(Pase et al. 2019), 
A $\beta 42 / A \beta 40$ (Chouraki et al. 2015) and NfL (Mattsson et al. 2019), only P-tau181 was associated with increased risk of AD dementia. These findings indicate that plasma P-tau181 could be used in clinical practice in primary care to identify individuals who would benefit from symptomatic treatment and in clinical trials to risk stratify asymptomatic participants and patients with MCI.

The finding that plasma P-tau181 correlated to $A \beta$ measured by $\left[{ }^{18} \mathrm{~F}\right]$ flutemetamol points to a link between metabolism of plasma P-tau181 and $A \beta$ build-up in the brain. The overall AUC to differentiate $A \beta$ - and $A \beta+$ individuals was 0.81 , which increased to 0.84 when combined with plasma $A \beta 42 / A \beta 40$. Our spline model analysis suggested that plasma P-tau181 started to increase around the time of $A \beta$ positivity, but levels of plasma P-tau181 appeared to continue to increase as $\mathrm{A} \beta$ accumulated (although our analysis was cross-sectional), as shown by the correlations with $\left[{ }^{18} \mathrm{~F}\right]$ flutemetamol even within the $\mathrm{A} \beta+$ subgroup.

This study confirms some aspects of plasma P-tau181 described previously, but also adds completely new evidence, including that plasma P-tau181 correlates to significant Tau PET uptake in several different meta-ROIs (corresponding to Braak tau stages), can be used for differential diagnosis of $\mathrm{AD}$ versus non- $\mathrm{AD}$ neurodegenerative diseases, and accurately predicts conversion to AD dementia in non-demented individuals with a higher accuracy than previously suggested plasma AD biomarkers including T-tau, A $\beta 42 / A \beta 40$ ratio and NfL.

\section{Limitations}

The study has several limitations. First, the non-AD neurodegenerative diseases group was relatively small and predominantly included patients with PD and PDD. Thus, our findings need to be replicated in larger and clinically more relevant patient groups, preferably also in a primary care setting. Second, even if Tau PET correlates closely with tau pathology in the brain(Smith et al. 2016, Smith et al. 2018), we did not present any actual neuropathology data, which may be required to elucidate to what degree plasma P-tau181 depends on the buildup of tau pathology versus other brain changes in AD. Finally, plasma P-tau181 was measured using a research-grade assay and a fully validated clinical-grade assay together with a certified reference material (CRM), see(Kuhlmann et al. 2017), must be by hand for implementation in routine clinical practice and establishment of universal cutoffs.

To conclude, plasma P-tau181 is a promising biomarker for diagnosis of AD and prognostication of clinical progression, and in drug trials to enrich eligible participants and potentially also to monitor biological effects of treatments. The NIA-AA research framework on $\mathrm{AD}$ (Jack et al. 2018) suggests that CSF P-tau181 can be used to indicate individual-specific tau status. Given the obvious benefit of being a blood test, together with the close correlations to CSF P-tau181, Tau PET load, AD diagnosis, and longitudinal conversion to $\mathrm{AD}$, we suggest that plasma P-tau181 can be used to identify tau status in future classification systems.

\section{ACKNOWLEDGMENTS}

Work at the authors' research center was supported by the European Research Council, the Swedish Research Council, the Knut and Alice Wallenberg foundation, the Marianne and Marcus Wallenberg foundation, the Strategic Research Area MultiPark (Multidisciplinary Research in Parkinson's disease) at Lund University, the Swedish Alzheimer Foundation, the Swedish Brain Foundation, The Parkinson foundation of Sweden, The Parkinson Research Foundation, the Skåne University Hospital Foundation, the Torsten Söderberg Foundation, the Swedish federal government under the ALF agreement, the Medical Faculty at Lund University, the Bundy Academy, and Region Skåne.

Doses of ${ }^{18} \mathrm{~F}$-flutemetamol injection were sponsored by GE Healthcare. The precursor of ${ }^{18} \mathrm{~F}$ flortaucipir was provided by AVID radiopharmaceuticals. 


\section{AUTHOR CONTRIBUTIONS}

\section{POTENTIAL CONFLICTS OF INTEREST}

Drs Janelidze, Palmqvist, Stomrud, XXX report no disclosures

NM has been a consultant for ADNI. RS has served as a (non-paid) consultant for Roche.

HZ has served at scientific advisory boards for Roche Diagnostics, Wave, Samumed and CogRx, has given lectures in symposia sponsored by Alzecure and Biogen, and is a co-founder of Brain Biomarker Solutions in Gothenburg AB, a GU Ventures-based platform company at the University of Gothenburg, all unrelated to the work presented in this paper.

KB has served as a consultant or at advisory boards for Alector, Biogen, CogRx, Lilly, MagQu, Novartis and Roche Diagnostics, and is a co-founder of Brain Biomarker Solutions in Gothenburg $\mathrm{AB}$, a GU Ventures-based platform company at the University of Gothenburg, all unrelated to the work presented in this paper.

$\mathrm{OH}$ has acquired research support (for the institution) from Roche, GE Healthcare, Biogen, AVID Radiopharmaceuticals and Euroimmun. In the past 2 years, he has received consultancy/speaker fees (paid to the institution) from Biogen and Roche. 


\section{FIGURE LEGENDS}

Figure 1. Association of plasma P-tau 181 with CSF P-tau181 and $\left[{ }^{18}\right.$ F $]$ flortaucipir PET in cohort 1. (A) Association between plasma P-tau181 and CSF P-tau181. (B) Association between plasma P-tau181 and $\left[{ }^{18} \mathrm{~F}\right]$ flortaucipir SUVR in temporal meta-ROI. (C) Voxel-wise regression analysis adjusted for age in a subcohort of cognitively unimpaired $(n=62)$ and patients with MCI $(\mathrm{n}=27)$ and AD dementia $(\mathrm{n}=35)$.

$\mathrm{A} \beta+=$ Amyloid- $\beta$-positive; $\mathrm{A} \beta$ - = Amyloid- $\beta$-negative, $\mathrm{CSF}=$ cerebrospinal fluid $; \mathrm{FWE}=$ familywise error; ROI = region of interest; $\mathrm{Rs}=$ Spearman correlation coefficient; $S U V R=$ standardized uptake values ratios.

Figure 2. Plasma P-tau181 and $\left[{ }^{18}\right.$ F $]$ flortaucipir retention in the Braak ROI in cohort 1. (A) Plasma concentrations of P-tau181 in individuals with abnormal $\left[{ }^{18} \mathrm{~F}\right]$ flortaucipir retention in the Braak I/II ROI (Braak I/II+ group), III/IV ROI (Braak III/IV+ group), V/VI ROI (Braak V/VI+ group) and normal retention in all Braak ROIs (FTP- group). $\left[{ }^{18} \mathrm{~F}\right]$ flortaucipir data were binarized based on the SUVR cutoff of 1.3(Ossenkoppele et al. 2018). The circles indicate individuals within the groups. In the Box-and-Whisker plot, the box ranges from the first to the third quartile, the vertical line represents the median of the diagnostic group and the whiskers indicate the range from the minimum to quartile 1 and from quartile 3 to the maximum excluding outliers. Outliers were defined as P-tau181 values less than quartile 1 or greater than quartile 3 by more than 1.5 times the interquartile range, and were shown as separate plotted points. The dotted line represents the median P-tau181 concentration in the FTP-group. P-values are from univariate general linear models adjusting for age and sex as described in the methods. (B) ROC curve analyses for predicting abnormal $\left[{ }^{18} \mathrm{~F}\right]$ flortaucipir status in the ITC, temporal meta-ROI and Braak I/II, III/IV and V/VI ROIs. The ROC curve and AUC of the Braak I/IV ROI overlapped with the Braak III/V ROI,

AUC $=$ area under the curve; $A \beta+=$ Amyloid $-\beta$ positive $; \mathrm{CI}=$ confidence interval; FTP $=$ $\left[{ }^{18}\right.$ F $]$ flortaucipir; ITC $=$ inferior temporal cortex; ROI $=$ region of interest ROC $=$ receiver operating characteristic.

Figure 3. Plasma P-tau181 in different diagnostic groups in cohort 1. (A) Plasma P-tau181 concentrations in $A \beta+$ and $A \beta$ - cognitively unimpaired (CU) and in $A \beta+M C I, A \beta+A D$ dementia and non-AD neurodegenerative diseases groups. The circles indicate individuals within the groups. In the Box-and-Whisker plot, the box ranges from the first to the third quartile, the vertical line represents the median of the diagnostic group and the whiskers indicate the range from the minimum to quartile 1 and from quartile 3 to the maximum excluding outliers. Outliers were defined as P-tau181 values less than quartile 1 or greater than quartile 3 by more than 1.5 times the interquartile range, and are shown as separate plotted points. The dotted line represents the median P-tau181 concentration in the $A \beta$ - cognitively unimpaired group. P-values are from univariate general linear models adjusting for age and sex as described in the methods. (B) ROC curve analyses for distinguishing AD dementia from non-AD neurodegenerative disorders. CSF P-tau181 and $\left[{ }^{18} \mathrm{~F}\right]$ flortaucipir data have been previously partly published and are shown for reference(Mattsson et al. 2018).

$\mathrm{AD}=$ Alzheimer disease $; \mathrm{AUC}=$ area under the curve; $\mathrm{A} \beta+=$ Amyloid $-\beta$ positive $; \mathrm{A} \beta-=$ Amyloid $\beta$ negative; $\mathrm{CI}=$ confidence interval; $\mathrm{CSF}=$ cerebrospinal fluid; $\mathrm{MCI}=$ Mild cognitive impairment; $\mathrm{ROC}=$ receiver operating characteristic $; \mathrm{ROI}=$ region of interest.

Figure 4. Plasma P-tau181 in cohort 2: differences between the diagnostic groups and associations with $\left[{ }^{18} \mathrm{~F}\right]$ flutemetamol PET and prediction of conversion to AD dementia. 
(A) Plasma P-tau181 in relation to global cortical $\left[{ }^{18} \mathrm{~F}\right]$ flutemetamol load. The solid lines is a fit from a spline model of P-tau181 on 18F-flutemetamol. The thick dotted line shows an a priori $\left[{ }^{18} \mathrm{~F}\right]$ flutemetamol threshold for A $\beta$ positivity (0.743 SUVR). The thin dotted lines indicate the $\left[{ }^{18} \mathrm{~F}\right]$ flutemetamol level where tau biomarkers are significantly increased from baseline. (B) Plasma P-tau 181 concentrations in $\mathrm{A} \beta+$ and $\mathrm{A} \beta$ - cognitively unimpaired (CU) and MCI patients. The circles indicate individuals within the groups. In the Box-and-Whisker plot, the box ranges from the first to the third quartile, the vertical line represents the median of the diagnostic group and the whiskers indicate the range from the minimum to quartile 1 and from quartile 3 to the maximum excluding outliers. Outliers were defined as P-tau181 values less than quartile 1 or greater than quartile 3 by more than 1.5 times the interquartile range, and are shown as separate plotted points. The dotted line represents the median P-tau181 concentration in the A $\beta$ - cognitively unimpaired group. P-values are from univariate general linear models adjusting for age and sex as described in the methods. (C) Survival curves for progression from cognitively unimpaired or MCI to AD dementia among participants with low (lowest tertile), intermediate (middle tertile) and high (highest tertile) baseline plasma P-tau181 levels. Adjustment were made for age, sex and years of educations.

$\mathrm{AD}=$ Alzheimer disease $; \mathrm{A} \beta+=$ Amyloid $-\beta$ positive $; \mathrm{A} \beta-=$ Amyloid $-\beta$ negative $; \mathrm{MCI}=$ Mild cognitive impairment; SUVR = standardized uptake values ratios. 
TABLES

Table 1. Demographic and clinical characteristics.

\begin{tabular}{|c|c|c|c|c|c|c|c|c|}
\hline & \multicolumn{4}{|c|}{ Cohort 1} & \multicolumn{4}{|c|}{ Cohort 2} \\
\hline & $\begin{array}{l}\mathrm{CU} \\
\mathrm{n}=64\end{array}$ & $\begin{array}{l}A \beta+M C I \\
n=28\end{array}$ & $\begin{array}{l}\mathbf{A} \boldsymbol{\beta}+\mathbf{A D} \\
\mathbf{n}=38\end{array}$ & $\begin{array}{l}\text { Non-AD diseases }{ }^{a} \\
\mathbf{n}=52\end{array}$ & $\begin{array}{l}A \beta-C U \\
n=126\end{array}$ & $\begin{array}{l}\mathbf{A} \boldsymbol{\beta}+\mathbf{C U} \\
\mathbf{n}=93\end{array}$ & $\begin{array}{l}\text { A } \beta-\text { MCI } \\
n=44\end{array}$ & $\begin{array}{l}\mathrm{A} \beta+\mathrm{MCI} \\
\mathrm{n}=\mathbf{8 1}\end{array}$ \\
\hline Age, years & $74(7)$ & $72(9)$ & $72(7)$ & $70(6)$ & $71(5)$ & $72(5)$ & $69(6)$ & $72(5)$ \\
\hline $\operatorname{Sex}$ F/M, $n$ & $33 / 31$ & $9 / 19$ & $17 / 21$ & $20 / 32$ & $78 / 48$ & $48 / 45$ & $13 / 31$ & $35 / 46$ \\
\hline Education, years b & $12(4)$ & $12(4)$ & $12(4)$ & $13(4)$ & $12(3)$ & $12(3)$ & $11(3)$ & $11(3)$ \\
\hline MMSE $^{\text {b }}$ & $29(1)$ & $26(3)$ & $20(5)$ & $25(5)$ & $29(1)$ & $29(1)$ & $28(2)$ & $27(2)$ \\
\hline CSF A $\beta 42, p g / m l$ & $508(188)$ & $330(105)$ & $290(97)$ & $517(240)$ & $798(191)$ & $400(125)$ & $799(205)$ & $356(131)$ \\
\hline CSF t-tau, $p g / m l$ & $369(124)$ & $592(164)$ & $594(207)$ & $317(144)$ & $292(72)$ & $363(195)$ & $303(93)$ & 489 (224) \\
\hline CSF P-tau181, $p g / m l$ & $154(86)$ & $378(168)$ & $420(217)$ & $115(92)$ & $98(30)$ & $170(138)$ & $96(29)$ & $287(191)$ \\
\hline $\begin{array}{l}\text { Plasma P-tau181, } \\
\mathrm{pg} / \mathrm{ml}\end{array}$ & $2.1(1.2)$ & $4.3(2.3)$ & $4.7(2.0)$ & $1.5(1.1)$ & $1.5(1.1)$ & $2.3(1.3)$ & $1.4(0.9)$ & $3.5(2.0)$ \\
\hline Plasma A $\beta 42, p g / m l^{\mathrm{b}}$ & N/A & N/A & N/A & N/A & $32(5)$ & $31(5)$ & $34(5)$ & $32(5)$ \\
\hline Plasma $A \beta 42 / A \beta 40^{b}$ & N/A & N/A & N/A & N/A & $0.068(0.008)$ & $0.064(0.008)$ & $0.067(0.008)$ & $0.063(0.006)$ \\
\hline Plasma t-tau, $p g / m l^{\text {a }}$ & N/A & N/A & N/A & N/A & $16(4)$ & $18(5)$ & $20(8)$ & $20(6)$ \\
\hline Plasma NfL, $p g / m l^{\text {a }}$ & N/A & N/A & N/A & N/A & $19(8)$ & $23(11)$ & $29(40)$ & $26(12)$ \\
\hline
\end{tabular}

Data are shown as mean (SD) unless otherwise specified. 
${ }^{\text {a }}$ Non-AD neurodegenerative diseases included 11 PD, 17 PDD, 6 PSP, 6 DLB, 6 CBS, 2 SD and 4 bvFTD patients.

${ }^{\mathrm{b}}$ In cohort 1 education was missing for 2 study participants; MMSE was missing for 6 study participants; CSF A $\beta 42$, A $\beta 42 / \mathrm{A} \beta 40$ and T-tau were missing for 2 study participants; $A \beta$ status was not available for 2 non-AD neurodegenerative diseases patients; in cohort 2 , education was missing for 4 study participants; CSF P-tau 181 and plasma NfL were missing for 1 study participant; plasma A $\beta 42$ was missing for 5 study participants.

$\mathrm{AD}=$ Alzheimer's disease dementia; $\mathrm{A} \beta+=$ Amyloid $-\beta$ positive; $\mathrm{A} \beta-=$ Amyloid- $\beta$ negative; bvFTD $=$ behavioral-variant frontotemporal dementia; $\mathrm{CBS}=$ corticobasal syndrome; $\mathrm{CU}=$ cognitively unimpaired; $\mathrm{DLB}=$ dementia with Lewy bodies; $\mathrm{F}=$ female; $\mathrm{M}=\mathrm{male} ; \mathrm{MCI}=$ mild cognitive impairment; MMSE = Mini Mental State Examinations; PD = Parkinson's disease; PDD = Parkinson's disease with dementia; PSP = progressive supranuclear palsy; $\mathrm{SD}=$ semantic dementia. 


\section{REFERENCES}

Armstrong, M. J., I. Litvan, A. E. Lang, T. H. Bak, K. P. Bhatia, B. Borroni, A. L. Boxer, D. W.

Dickson, M. Grossman, M. Hallett, K. A. Josephs, A. Kertesz, S. E. Lee, B. L. Miller, S. G. Reich, D. E. Riley, E. Tolosa, A. I. Troster, M. Vidailhet and W. J. Weiner (2013). "Criteria for the diagnosis of corticobasal degeneration." Neurology 80(5): 496-503.

Beach, T. G., S. E. Monsell, L. E. Phillips and W. Kukull (2012). "Accuracy of the clinical diagnosis of Alzheimer disease at National Institute on Aging Alzheimer Disease Centers, 20052010." J Neuropathol Exp Neurol 71(4): 266-273.

Blennow, K., H. Hampel, M. Weiner and H. Zetterberg (2010). "Cerebrospinal fluid and plasma biomarkers in Alzheimer disease." Nat Rev Neurol 6(3): 131-144.

Cho, H., J. Y. Choi, M. S. Hwang, Y. J. Kim, H. M. Lee, H. S. Lee, J. H. Lee, Y. H. Ryu, M. S. Lee and C. H. Lyoo (2016). "In vivo cortical spreading pattern of tau and amyloid in the Alzheimer disease spectrum." Ann Neurol 80(2): 247-258.

Chouraki, V., A. Beiser, L. Younkin, S. R. Preis, G. Weinstein, O. Hansson, I. Skoog, J. C. Lambert, R. Au, L. Launer, P. A. Wolf, S. Younkin and S. Seshadri (2015). "Plasma amyloid-beta and risk of Alzheimer's disease in the Framingham Heart Study." Alzheimers Dement 11(3): 249257 e241.

Couchie, D., C. Mavilia, I. S. Georgieff, R. K. Liem, M. L. Shelanski and J. Nunez (1992). "Primary structure of high molecular weight tau present in the peripheral nervous system." Proc Natl Acad Sci U S A 89(10): 4378-4381.

Gelb, D. J., E. Oliver and S. Gilman (1999). "Diagnostic criteria for Parkinson disease." Arch Neurol 56(1): 33-39.

Hahn, A., M. Schain, M. Erlandsson, P. Sjolin, G. M. James, O. T. Strandberg, D. Hagerstrom, R. Lanzenberger, J. Jogi, T. G. Olsson, R. Smith and O. Hansson (2017). "Modeling Strategies for Quantification of In Vivo (18)F-AV-1451 Binding in Patients with Tau Pathology." J Nucl Med 58(4): 623-631. 
Hansson, O., H. Zetterberg, P. Buchhave, E. Londos, K. Blennow and L. Minthon (2006).

"Association between CSF biomarkers and incipient Alzheimer's disease in patients with mild cognitive impairment: a follow-up study." Lancet Neurol 5(3): 228-234.

Hoglinger, G. U., G. Respondek, M. Stamelou, C. Kurz, K. A. Josephs, A. E. Lang, B.

Mollenhauer, U. Muller, C. Nilsson, J. L. Whitwell, T. Arzberger, E. Englund, E. Gelpi, A. Giese,

D. J. Irwin, W. G. Meissner, A. Pantelyat, A. Rajput, J. C. van Swieten, C. Troakes, A. Antonini, K.

P. Bhatia, Y. Bordelon, Y. Compta, J. C. Corvol, C. Colosimo, D. W. Dickson, R. Dodel, L.

Ferguson, M. Grossman, J. Kassubek, F. Krismer, J. Levin, S. Lorenzl, H. R. Morris, P. Nestor, W.

H. Oertel, W. Poewe, G. Rabinovici, J. B. Rowe, G. D. Schellenberg, K. Seppi, T. van Eimeren, G.

K. Wenning, A. L. Boxer, L. I. Golbe, I. Litvan and P. S. P. S. G. Movement Disorder Societyendorsed (2017). "Clinical diagnosis of progressive supranuclear palsy: The movement disorder society criteria." Mov Disord 32(6): 853-864.

Jack, C. R., Jr., D. A. Bennett, K. Blennow, M. C. Carrillo, B. Dunn, S. B. Haeberlein, D. M. Holtzman, W. Jagust, F. Jessen, J. Karlawish, E. Liu, J. L. Molinuevo, T. Montine, C. Phelps, K. P. Rankin, C. C. Rowe, P. Scheltens, E. Siemers, H. M. Snyder, R. Sperling and Contributors (2018). "NIA-AA Research Framework: Toward a biological definition of Alzheimer's disease." Alzheimers Dement 14(4): 535-562.

Kuhlmann, J., U. Andreasson, J. Pannee, M. Bjerke, E. Portelius, A. Leinenbach, T. Bittner, M. Korecka, R. G. Jenkins, H. Vanderstichele, E. Stoops, P. Lewczuk, L. M. Shaw, I. Zegers, H.

Schimmel, H. Zetterberg, K. Blennow and I. W. G. o. S. o. C. proteins (2017). "CSF Abeta1-42 - an excellent but complicated Alzheimer's biomarker - a route to standardisation." Clin Chim Acta 467: 27-33.

Litvan, I., Y. Agid, D. Calne, G. Campbell, B. Dubois, R. C. Duvoisin, C. G. Goetz, L. I. Golbe, J. Grafman, J. H. Growdon, M. Hallett, J. Jankovic, N. P. Quinn, E. Tolosa and D. S. Zee (1996). "Clinical research criteria for the diagnosis of progressive supranuclear palsy (Steele-RichardsonOlszewski syndrome): report of the NINDS-SPSP international workshop." Neurology 47(1): 1-9. 
Maass, A., S. Landau, S. L. Baker, A. Horng, S. N. Lockhart, R. La Joie, G. D. Rabinovici, W. J. Jagust and I. Alzheimer's Disease Neuroimaging (2017). "Comparison of multiple tau-PET measures as biomarkers in aging and Alzheimer's disease." Neuroimage 157: 448-463.

Mattsson, N., N. C. Cullen, U. Andreasson, H. Zetterberg and K. Blennow (2019). "Association Between Longitudinal Plasma Neurofilament Light and Neurodegeneration in Patients With Alzheimer Disease." JAMA Neurol.

Mattsson, N., P. S. Insel, S. Palmqvist, E. Stomrud, D. van Westen, L. Minthon, H. Zetterberg, K. Blennow and O. Hansson (2016). "Increased amyloidogenic APP processing in APOE varepsilon4negative individuals with cerebral beta-amyloidosis." Nat Commun 7: 10918.

Mattsson, N., M. Scholl, O. Strandberg, R. Smith, S. Palmqvist, P. S. Insel, D. Hagerstrom, T.

Ohlsson, H. Zetterberg, J. Jogi, K. Blennow and O. Hansson (2017). "(18)F-AV-1451 and CSF Ttau and P-tau as biomarkers in Alzheimer's disease." EMBO Mol Med 9(9): 1212-1223.

Mattsson, N., R. Smith, O. Strandberg, S. Palmqvist, M. Scholl, P. S. Insel, D. Hagerstrom, T.

Ohlsson, H. Zetterberg, K. Blennow, J. Jogi and O. Hansson (2018). "Comparing (18)F-AV-1451 with CSF t-tau and p-tau for diagnosis of Alzheimer disease." Neurology 90(5): e388-e395.

Mattsson, N., H. Zetterberg, S. Janelidze, P. S. Insel, U. Andreasson, E. Stomrud, S. Palmqvist, D. Baker, C. A. Tan Hehir, A. Jeromin, D. Hanlon, L. Song, L. M. Shaw, J. Q. Trojanowski, M. W. Weiner, O. Hansson, K. Blennow and A. Investigators (2016). "Plasma tau in Alzheimer disease." Neurology 87(17): 1827-1835.

Mielke, M. M., C. E. Hagen, J. Xu, X. Chai, P. Vemuri, V. J. Lowe, D. C. Airey, D. S. Knopman, R. O. Roberts, M. M. Machulda, C. R. Jack, Jr., R. C. Petersen and J. L. Dage (2018). "Plasma phospho-tau181 increases with Alzheimer's disease clinical severity and is associated with tau- and amyloid-positron emission tomography." Alzheimers Dement 14(8): 989-997.

Ossenkoppele, R., W. J. Jansen, G. D. Rabinovici, D. L. Knol, W. M. van der Flier, B. N. van Berckel, P. Scheltens, P. J. Visser, P. E. T. S. G. Amyloid, S. C. Verfaillie, M. D. Zwan, S. M. Adriaanse, A. A. Lammertsma, F. Barkhof, W. J. Jagust, B. L. Miller, H. J. Rosen, S. M. Landau, 
V. L. Villemagne, C. C. Rowe, D. Y. Lee, D. L. Na, S. W. Seo, M. Sarazin, C. M. Roe, O. Sabri, H. Barthel, N. Koglin, J. Hodges, C. E. Leyton, R. Vandenberghe, K. van Laere, A. Drzezga, S. Forster, T. Grimmer, P. Sanchez-Juan, J. M. Carril, V. Mok, V. Camus, W. E. Klunk, A. D. Cohen, P. T. Meyer, S. Hellwig, A. Newberg, K. S. Frederiksen, A. S. Fleisher, M. A. Mintun, D. A. Wolk, A. Nordberg, J. O. Rinne, G. Chetelat, A. Lleo, R. Blesa, J. Fortea, K. Madsen, K. M. Rodrigue and D. J. Brooks (2015). "Prevalence of amyloid PET positivity in dementia syndromes: a metaanalysis." JAMA 313(19): 1939-1949.

Ossenkoppele, R., G. D. Rabinovici, R. Smith, H. Cho, M. Scholl, O. Strandberg, S. Palmqvist, N. Mattsson, S. Janelidze, A. Santillo, T. Ohlsson, J. Jogi, R. Tsai, R. La Joie, J. Kramer, A. L. Boxer, M. L. Gorno-Tempini, B. L. Miller, J. Y. Choi, Y. H. Ryu, C. H. Lyoo and O. Hansson (2018). "Discriminative Accuracy of [18F]flortaucipir Positron Emission Tomography for Alzheimer Disease vs Other Neurodegenerative Disorders." JAMA 320(11): 1151-1162.

Ossenkoppele, R., R. Smith, T. Ohlsson, O. Strandberg, N. Mattsson, P. S. Insel, S. Palmqvist and O. Hansson (2019). "Associations between tau, Abeta, and cortical thickness with cognition in Alzheimer disease." Neurology 92(6): e601-e612.

Palmqvist, S., P. S. Insel, H. Zetterberg, K. Blennow, B. Brix, E. Stomrud, I. Alzheimer's Disease Neuroimaging, F. s. Swedish Bio, N. Mattsson and O. Hansson (2019). "Accurate risk estimation of beta-amyloid positivity to identify prodromal Alzheimer's disease: Cross-validation study of practical algorithms." Alzheimers Dement 15(2): 194-204.

Palmqvist, S., S. Janelidze, E. Stomrud, H. Zetterberg, J. Karl, K. Zink, T. Bittner, N. Mattsson, U. Eichenlaub, K. Blennow and O. Hansson (2019). "Performance of Fully Automated Plasma Assays as Screening Tests for Alzheimer Disease-Related beta-Amyloid Status." JAMA Neurol.

Palmqvist, S., M. Scholl, O. Strandberg, N. Mattsson, E. Stomrud, H. Zetterberg, K. Blennow, S. Landau, W. Jagust and O. Hansson (2017). "Earliest accumulation of beta-amyloid occurs within the default-mode network and concurrently affects brain connectivity." Nat Commun 8(1): 1214 . 
Palmqvist, S., H. Zetterberg, K. Blennow, S. Vestberg, U. Andreasson, D. J. Brooks, R. Owenius, D. Hagerstrom, P. Wollmer, L. Minthon and O. Hansson (2014). "Accuracy of brain amyloid detection in clinical practice using cerebrospinal fluid beta-amyloid 42: a cross-validation study against amyloid positron emission tomography." JAMA Neurol 71(10): 1282-1289.

Pase, M. P., A. S. Beiser, J. J. Himali, C. L. Satizabal, H. J. Aparicio, C. DeCarli, G. Chene, C. Dufouil and S. Seshadri (2019). "Assessment of Plasma Total Tau Level as a Predictive Biomarker for Dementia and Related Endophenotypes." JAMA Neurol.

Petersen, R. C. (2004). "Mild cognitive impairment as a diagnostic entity." J Intern Med 256(3): 183-194.

Scholl, M., S. N. Lockhart, D. R. Schonhaut, J. P. O'Neil, M. Janabi, R. Ossenkoppele, S. L. Baker, J. W. Vogel, J. Faria, H. D. Schwimmer, G. D. Rabinovici and W. J. Jagust (2016). "PET Imaging of Tau Deposition in the Aging Human Brain." Neuron 89(5): 971-982.

Scholl, M., A. Maass, N. Mattsson, N. J. Ashton, K. Blennow, H. Zetterberg and W. Jagust (2018). "Biomarkers for tau pathology." Mol Cell Neurosci.

Smith, R., A. Puschmann, M. Scholl, T. Ohlsson, J. van Swieten, M. Honer, E. Englund and O. Hansson (2016). "18F-AV-1451 tau PET imaging correlates strongly with tau neuropathology in MAPT mutation carriers." Brain 139(Pt 9): 2372-2379.

Smith, R., M. Wibom, D. Pawlik, E. Englund and O. Hansson (2018). "Correlation of In Vivo [18F]Flortaucipir With Postmortem Alzheimer Disease Tau Pathology." JAMA Neurol.

Tatebe, H., T. Kasai, T. Ohmichi, Y. Kishi, T. Kakeya, M. Waragai, M. Kondo, D. Allsop and T. Tokuda (2017). "Quantification of plasma phosphorylated tau to use as a biomarker for brain Alzheimer pathology: pilot case-control studies including patients with Alzheimer's disease and down syndrome." Mol Neurodegener 12(1): 63.

Van Essen, D. C. (2005). "A Population-Average, Landmark- and Surface-based (PALS) atlas of human cerebral cortex." Neuroimage 28(3): 635-662. 
Yang, C. C., M. J. Chiu, T. F. Chen, H. L. Chang, B. H. Liu and S. Y. Yang (2018). "Assay of Plasma Phosphorylated Tau Protein (Threonine 181) and Total Tau Protein in Early-Stage Alzheimer's Disease." J Alzheimers Dis 61(4): 1323-1332.

Zetterberg, H., D. Wilson, U. Andreasson, L. Minthon, K. Blennow, J. Randall and O. Hansson (2013). "Plasma tau levels in Alzheimer's disease." Alzheimers Res Ther 5(2): 9. 